\title{
A new ex vivo method to evaluate the performance of candidate MRI contrast agents: a proof-of-concept study
}

Ana Paula Candiota ${ }^{1,2,3}$, Milena Acosta ${ }^{1,2}$, Rui Vasco Simões ${ }^{2,1}$, Teresa Delgado-Goñi ${ }^{2,1}$, Silvia Lope-Piedrafita ${ }^{4,1}$, Ainhoa Irure ${ }^{5,1}$, Marco Marradi ${ }^{1,5}$, Oscar Bomati-Miguel ${ }^{6}$, Nuria Miguel-Sancho ${ }^{7,1}$, Ibane Abasolo ${ }^{1,8}$, Simó Schwartz Jr J,8, Jesús Santamaria ${ }^{5,1}$, Soledad Penadés ${ }^{5,1}$ and Carles Arús 2,1,3* $^{*}$

\begin{abstract}
Background: Magnetic resonance imaging (MRI) plays an important role in tumor detection/diagnosis. The use of exogenous contrast agents (CAs) helps to improve the discrimination between lesion and neighbouring tissue, but most of the currently available CAs are non-specific. Assessing the performance of new, selective CAs requires exhaustive assays and large amounts of material. Accordingly, in a preliminary screening of new CAs, it is important to choose candidate compounds with good potential for in vivo efficiency. This screening method should reproduce as close as possible the in vivo environment. In this sense, a fast and reliable method to select the best candidate CAs for in vivo studies would minimize time and investment cost, and would benefit the development of better CAs.

Results: The post-mortem ex vivo relative contrast enhancement (RCE) was evaluated as a method to screen different types of CAs, including paramagnetic and superparamagnetic agents. In detail, sugar/gadolinium-loaded gold nanoparticles (Gd-GNPs) and iron nanoparticles (SPIONs) were tested. Our results indicate that the post-mortem ex vivo RCE of evaluated CAs, did not correlate well with their respective in vitro relaxivities. The results obtained with different Gd-GNPs suggest that the linker length of the sugar conjugate could modulate the interactions with cellular receptors and therefore the relaxivity value. A paramagnetic CA (GNP (E_2)), which performed best among a series of Gd-GNPs, was evaluated both ex vivo and in vivo. The ex vivo RCE was slightly worst than gadoterate meglumine ( $201.9 \pm 9.3 \%$ versus $237 \pm 14 \%$, respectively), while the in vivo RCE, measured at the time-to-maximum enhancement for both compounds, pointed to GNP E_2 being a better CA in vivo than gadoterate meglumine. This is suggested to be related to the nanoparticule characteristics of the evaluated GNP.

Conclusion: We have developed a simple, cost-effective relatively high-throughput method for selecting CAs for in vivo experiments. This method requires approximately 800 times less quantity of material than the amount used for in vivo administrations.
\end{abstract}

Keywords: Magnetic nanoparticles, Magnetic resonance imaging, Contrast media, ex vivo screening

\footnotetext{
*Correspondence: carles.arus@uab.es

${ }^{2}$ Departament de Bioquímica i Biologia Molecular, Unitat de Bioquímica de Biociències, Edifici Cs, Universitat Autònoma de Barcelona, Cerdanyola del Vallès, Barcelona 08193 Spain

${ }^{1}$ Centro de Investigación Biomédica en Red - Bioingeniería, Biomateriales y Nanomedicina (CIBER-BBN), Spain

Full list of author information is available at the end of the article
}

\section{Biomed Central}

(c) 2014 Candiota et al.; licensee BioMed Central Ltd. This is an Open Access article distributed under the terms of the Creative Commons Attribution License (http://creativecommons.org/licenses/by/2.0), which permits unrestricted use, distribution, and reproduction in any medium, provided the original work is properly credited. The Creative Commons Public Domain Dedication waiver (http://creativecommons.org/publicdomain/zero/1.0/) applies to the data made available in this article, unless otherwise stated. 


\section{Background}

Magnetic resonance imaging (MRI) plays an important role in early tumor detection and diagnosis. The use of exogenous contrast agents (CAs) $[1,2]$ is helpful to improve the discrimination between lesion and neighbouring tissue.

Contrast agents (CAs) can enhance tissue contrast by shortening longitudinal $\left(\mathrm{T}_{1}\right)$ and transverse $\left(\mathrm{T}_{2}\right)$ relaxation times of water. The magnitude of this effect is measured as relaxivity $\left(r_{1}\right.$ or $\left.r_{2}\right)$ and it is related to the efficacy of the CAs in accelerating relaxation. This parameter can be modulated by many factors, such as protein binding, chemical structure and dynamic processes (e.g. water exchange kinetics, first and second coordination sphere hydration, rotational tumbling rates) $[3,4]$. When administered at equivalent doses, compounds with high relaxivity values could generate greater contrast than compounds with lower relaxivity, and therefore less amount is required to generate the same effect. On the other hand, compounds with low in vitro relaxivity but with specific binding to some cellular/molecular targets can also have potential application as CAs in MRI [5]. Most of the currently available extracellular CAs are non-specific and less efficient than predicted by theory [6], and most of the in vitro strategies used to evaluate their performance are not able to reproduce the in vivo conditions that could modify their ability to generate contrast [5,7-9].

Analyzing the in vivo performance of a new candidate CA involves exhaustive assays and requires large amounts of material, not always available at early stages of product development. Accordingly, a preliminary screening of new CAs should ideally choose candidates with good in vivo potential, in order to minimize time and cost invested. In this process, one also needs to consider the ability of the $\mathrm{CA}$ to reach the target tissue and to interact with the extracellular matrix or substances released by cells. In this sense, the standard in vitro method currently used for evaluating CA performance, relaxivity calculation [10], may not be a good predictor of in vivo performance. Up to date, there is not any "ideal" ex vivo method, although some have been described $[11,12]$. The use of a fast and reliable method to select CAs from a range of candidates, reproducing as close as possible the biological environment and requiring small amounts of material, would benefit the development of new and better CAs.

The purpose of this work was to design and evaluate a simple ex vivo method to assess the performance of new CAs for preclinical brain tumor detection using minimal amounts of material. This method should be a better predictor of in vivo performance than classic in vitro relaxivity measurements. To validate it, we have tested novel paramagnetic Gd-based gold nanoparticles (Gd-GNPs) (Figure 1) [7,13] as well as superparamagnetic iron oxide nanoparticles (SPIONs) [14]. The Gd-GNPs incorporate several copies of sugars (which confer biocompatibility
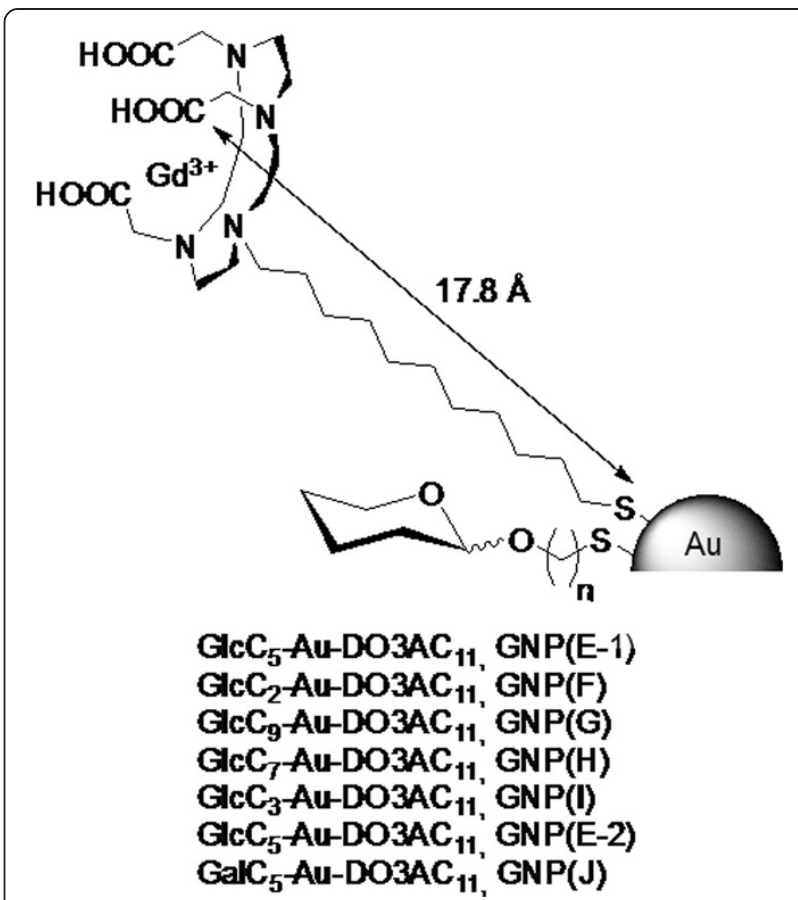

Figure 1 Short name: Schematic representation of the paramagnetic Gd-chelate containing gold glyconanoparticle (Gd-GNPs). Detailed legend: Schematic representation of the paramagnetic Gd-chelate containing gold glyconanoparticle (Gd-GNPs) tested in this work. The gold core (Au) is coated with Gd:D03A derivatives and self-assembled monolayer of saccharide conjugates. Thiol-ended alkane linkers are used as spacers to attach the conjugates to the gold core. Figure not drawn to scale. Glc = glucose. See also Table 1, text and reference [7] for further details.

and water solubility) and Gd complexes. The multivalent presentation of sugars in these Gd-GNPs gives them targeting properties, enhancing their avidity for carbohydratebinding receptors at the cell surface and could also modulate their cellular uptake.

Iron oxide, and specifically iron oxide nanoparticles, are the most investigated material for negative $\mathrm{CA}$ in biomedicine because of its high magnetic moment, chemical stability in physiological conditions and low toxicity $[15,16]$. Further engineering of these nanoparticles should improve their performance, making them more selective and thus more efficient [17-20]. Nevertheless, some of the modifications introduced in the iron nanoparticles can alter their superparamagnetic properties (and, hence, the contrast effect obtained) which should be evaluated before going further in nanoparticle development.

\section{Results}

Post mortem ex vivo RCE analysis and in vitro $1.4 \mathrm{~T}$ and 7.0 $\mathrm{T}$ relaxivity measurements were performed for all nanoparticles, while in vivo measurements in mice were restricted to the best performing Gd-GNP candidate. 


\section{In vitro relaxivity studies}

The $r_{1}$ relaxivity values were measured for positive contrast agents, both at 1.4 and 7.0 T, and are listed in Table 1. As expected, $\mathrm{r}_{1}$ decreases with increasing magnetic field [21]. At 1.4 $\mathrm{T}$, all the Gd-GNPs relaxivity values were higher than gadoterate meglumine, whereas at $7.0 \mathrm{~T}$ a higher dispersion was observed, with values both above and below gadoterate meglumine. It should be noted that the value obtained in vitro at 7.0 $\mathrm{T}$ for gadoterate meglumine (one of the gold standards for Gd-containing CAs), is in good agreement with literature values obtained in vivo for Gd-DTPA at $6.3 \mathrm{~T}\left(3.0 \mathrm{mM}^{-1} \mathrm{~s}^{-1}\right)$ [22], although at slightly different temperature $\left(23^{\circ} \mathrm{C}\right.$ in vitro and $37^{\circ} \mathrm{C}$ in vivo).

\section{Ex vivo post mortem $\mathrm{MRI}$ analysis}

A representative $T_{1}$-weighted image for Gd-GNP (E_2), as well as typical regions of interest (ROIs) selected for analysis, are shown in Figure $2 \mathrm{~A}$ and $2 \mathrm{~B}$. The contrast enhancement obtained with this method clearly varies depending on the Gd-GNP investigated (Figure 2C). All Gd-GNPs investigated produced significantly lower contrast enhancement compared with gadoterate meglumine $(236.7 \pm 8.3 \%$ with respect to contralateral RCE, Table 1). The only exception was GNP (F) which was lower but not significantly. Among all GNPs, the GNP (E_2) presented the highest contrast enhancement (201.9 \pm 9.3\%). These results could be partially explained due to the differences in $r_{1}$ and $r_{2} / r_{1}$ (Table 1) among the different CA. All GNPs but GNP (E_2) presented $r_{2} / r_{1}$ ranging from 5.00 to 11.06 , clearly higher than the $r_{2} / r_{1}$ ratio measured for gadoterate meglumine (1.70). On the other hand, the $r_{2} / r_{1}$ ratio for GNP (E_2) was of 1.77. In addition, these results suggest that the sugar linker length could play a role in the results: GNPs with a C5 linker for sugars (GlcC5 (GNP E_1), GalC5 (GNP J)) tend to provide higher positive contrast enhancement (\%RCE), when the chelating DO3A linker is C11 long. Still, GlcC2 (GNP F) also produced good results.

The ex vivo post-mortem MRI method was also successfully applied to iron-oxide nanoparticles. The results obtained have been summarized in the supplementary material (Section 2).

\section{In vivo MRI analysis}

$\mathrm{T}_{2}$-weighted images demonstrated the expected, predictable increase in tumor size and the overall morphology of a high grade glial tumor at 10 days post-inoculation of GL261 cells (Figure 3). The most consistent imaging finding in these tumors is the enhancement after contrast administration on post-contrast $\mathrm{T}_{1}$-weighted images. In this case, both contrast agents evaluated in vivo (gadoterate meglumine and GNP (E_2)) showed positive contrast enhancement on $\mathrm{T}_{1}$-weighted images (Figure 3 , $\mathrm{T}_{1}$-ref vs. $\mathrm{T}_{1}$-max, and Figure 4 ).
Following the same trend observed in the ex vivo postmortem studies, the GNP (E_2) showed a significantly $(\mathrm{p}<0.05)$ worse performance in GL261 tumors (RCE $107.9 \pm 5.9 \%)$ in comparison with gadoterate meglumine $(113.1 \pm 2.5 \%)$ when they were measured both at $2 \mathrm{~min}$ $56 \mathrm{sec}$ post administration (corresponding to the time of maximum enhancement of gadoterate meglumine, Figure 4). The discrepancy between the ex vivo postmortem studies (Table 1) and the information depicted in Figure 3 (RCE maps) is related to the time for maximum enhancement of each CA, which is significantly longer for GNP (E_2) (about $8 \mathrm{~min}$ ) than for gadoterate meglumine (about $3 \mathrm{~min})(\mathrm{p}<0.001)$, (Figure 4). If we take into account RCE at time of maximum enhancement for each CA (Figure 5), the GNP (E_2) outperforms gadoterate meglumine: RCE of $124.9 \pm 8.3$ and $113.1 \pm 2.5 \%$, respectively. In vivo imaging results were complemented by Induced Coupled Plasma-Mass Spectrometry (ICP-MS) measurements of gold content in tumor and normal brain (Figure 6). Indeed, Gd-GNP biodistribution assays after $24 \mathrm{~h}$ demonstrated that mouse brain tumors administered with GNP (E-2) accumulated almost ten times more CA than normal brain, and nearly twice the amount in circulating blood.

Renal and hepatic function parameters were also measured from the blood of animals administered with nanoparticles in vivo, including glucose, total proteins, albumin, creatinine, urea, total bilirrubine, aspartate aminotransferase (AST) and alanine aminotransferase (ALT)). Histopathological studies were also conducted, $24 \mathrm{~h}$ and 14 days post-administration of the CAs. No significant changes were found in any of the blood biochemical parameters analyzed in mice administered with GNP (E_1 or E_2) compared to control mice (only vehicle) as shown in Additional file 1: Table S1. Moreover, values for the three groups of mice remained within reference values $[23,24]$. Similarly, no alterations were found in histological sections of relevant organs such as liver, spleen and lungs (Additional file 1: Figure S2). Overall, these data suggest that the nanoparticles were non toxic to the animals in the time frame investigated although more detailed toxicological studies would be required in order to confirm (or refute) this assertion.

\section{Pharmacological parameters for CA tumor perfusion}

Table 2 and Figure 7 summarize \% of RCE ex vivo, \% of $\mathrm{RCE}$ in vivo, $\mathrm{K}_{\mathrm{ep}}$ (exchange rate constant) and IAUC (initial area under the curve) for the gadoterate meglumine and the GNP (E_2), which had a full evaluation in vitro, ex vivo and in vivo.

\section{Discussion}

Gadolinium complexes are widely used as positive CA in clinical MRI examinations. Besides the well known 
Table $1 \mathrm{Gd}$-GNPs studied, percentage of Gd (in $\mathrm{g}$ of Gd per $100 \mathrm{~g}$ of nanoparticle), relaxivity values $\left(\mathbf{r}_{1}, \mathbf{r}_{\mathbf{2}}\right.$ and $\left.\mathbf{r}_{2} / \mathbf{r}_{1}\right)$ and relative contrast enhancement (RCE) values

\begin{tabular}{|c|c|c|c|c|c|c|c|}
\hline $\begin{array}{l}\text { Contrast agent Gd-GNP } \\
\text { (Glyco-Au-D03A:Gd) }^{\mathrm{a}}\end{array}$ & $\begin{array}{l}\% \mathrm{Gd} \text { (g of Gd per } 100 \mathrm{~g} \\
\text { of nano particle) }\end{array}$ & $\begin{array}{c}1.4 \mathrm{Tr}_{1} \text { relaxivity } \\
\left(\mathrm{s}^{-1} \mathrm{mM}^{-1}\right) \text { (in vitro) }(\mathrm{n}=3)\end{array}$ & $\begin{array}{c}7.0 \mathrm{~T} r_{1} \text { relaxivity } \\
\left(\mathrm{s}^{1} \mathrm{mM}^{-1}\right) \text { (in vitro) }(\mathrm{n}=3)\end{array}$ & $\begin{array}{c}7.0 \mathrm{~T}_{2} \text { relaxivity }\left(\mathrm{s}^{-1} \mathrm{mM}^{-1}\right) \\
\text { (in vitro) }(\mathrm{n}=3)\end{array}$ & $\begin{array}{l}7.0 \mathrm{~T} \\
\mathrm{r}_{2} / \mathrm{r}_{1}\end{array}$ & $\begin{array}{l}\text { \% RCE postmorten } \\
\text { (ex vivo) }(n=3)\end{array}$ & $\begin{array}{c}\% \text { RCE (in vivo**) } \\
(\mathrm{n}=3)\end{array}$ \\
\hline GlcC5-DO3AC11 (GNP (E_1)) & $4.7 \pm 0.1$ & $7.4 \pm 0.7$ & $2.7 \pm 0.40$ & $20.2 \pm 0.11$ & 7.48 & $182.8 \pm 10.7^{*}$ & NM \\
\hline GlcC2-DO3AC11 (GNP (F)) & $3.4 \pm 0.2$ & $7.1 \pm 0.9$ & $2.9 \pm 0.04$ & $14.5 \pm 2.37$ & 5.00 & $185.4 \pm 17.2$ & NM \\
\hline GlcC9-DO3AC11 (GNP (G)) & $3.2 \pm 0.2$ & $7.5 \pm 0.6$ & $1.6 \pm 0.15$ & $12.7 \pm 0.24$ & 7.94 & $168.7 \pm 7.74^{*}$ & NM \\
\hline GlcC7-DO3AC11 (GNP (H)) & $4.1 \pm 0.2$ & $7.1 \pm 0.9$ & $1.6 \pm 0.36$ & $11.2 \pm 0.21$ & 7.00 & $143.6 \pm 8.0^{*}$ & NM \\
\hline GlcC3-DO3AC11 (GNP (I)) & $3.3 \pm 0.2$ & $6.3 \pm 0.7$ & $1.8 \pm 0.07$ & $19.9 \pm 1.43$ & 11.06 & $141.5 \pm 2.5$ & NM \\
\hline GlcC5-DO3AC11 (GNP (E_2) $)^{b}$ & $7.0 \pm 0.2$ & $11.5 \pm 0.1$ & $8.2 \pm 0.37$ & $14.5 \pm 0.66$ & 1.77 & $201.9 \pm 9.3^{*}$ & $124.9 \pm 8.3^{*}$ \\
\hline GlcC5-DO3AC11 (GNP (J)) & $5.0 \pm 0.2$ & $8.1 \pm 0.3$ & $4.6 \pm 0.24$ & $24.4 \pm 0.34$ & 5.30 & $169.1 \pm 14.1^{*}$ & NM \\
\hline Gadoterate meglumine & NA & $3.10^{c} / 3.5^{d}$ & $2.1 \pm 0.42$ & $3.58 \pm 0.02$ & 1.70 & $236.7 \pm 8.3$ & $113.1 \pm 2.5$ \\
\hline
\end{tabular}

\section{Detailed legend:}

a) $\mathrm{Glc}=$ glucose, Gal = galactose. $\mathrm{C}$ (number) after the saccharide and the DO3A indicates the number of carbon atoms of the linkers.

b) GNP (E_2) was obtained from GNP (E_1) after further incubation with a solution of DO3AC11-Gd complex (4 equiv.) during 44 hours as described in [31]

c) Values taken from [7].

d) Values taken from [58].
${ }^{*} p<0.05$ versus gadoterate meglumine.

** Mean of RCE values ( $n=9$ independent measures for 3 animals) measured at the maximum enhancement time, for each of the CA studied in vivo.

$N M$, not measured. NA, not applicable.

For each value mean and standard deviation are shown 


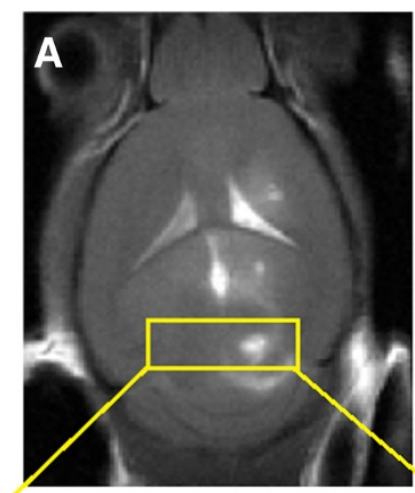

B

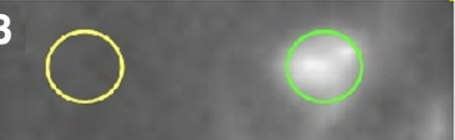

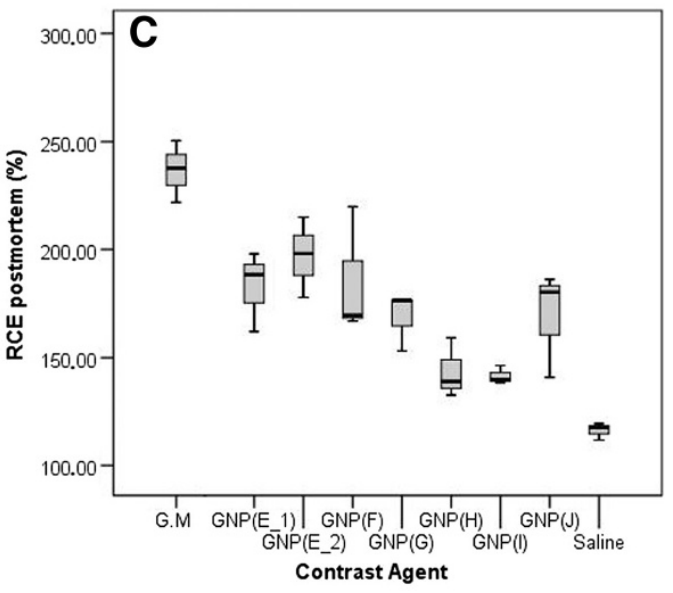

Contrast Agent

Figure 2 Short name: Ex vivo MRI and example of Gd-GNPs evaluated; Boxplot of relative contrast enhancement. Detailed legend: A) Representative ex vivo $T_{1}$-weighted image and example of ROls used (manually drawn as circles, green (ipsilateral) and yellow (contralateral) lines) for signal enhancement calculation for GNP (E_2). B) Enlarged central row (white discontinuous rectangle) C) Boxplot of relative contrast enhancement for saline solution, gadoterate meglumine (G.M.) and GNPs obtained from ROls of all CAs studied. RCE was calculated using Equation 1. The limits of the box represent the quartiles $1(\mathrm{Q} 1)$ and $3(\mathrm{Q} 3)$ of the distribution, the central line corresponds to the median (quartile 2) and the whiskers represent the maximum and minimum value in each distribution. The data represented were included in the range [Q1-1.5 IQR - Q3 + 1.5 IQR], being IQR the interquartile range.

association of these agents with nephrogenic systemic fibrosis [25], they are non specific, i.e. able to detect anatomical and morphological changes but not a specific pathology. Developing contrast agents with specific targeting properties would have a significant clinical impact. One of the ways to approach this goal is to develop nano-platforms for attaching simultaneously a $\mathrm{CA}$ and targeting molecules forming nano-sized contrast agents [26-30].

The new positive CAs tested in this work are gold glyconanoparticles (GNPs) coated with multiple copies of sugars and Gd-complexes (Gd-GNPs) (Figure 1

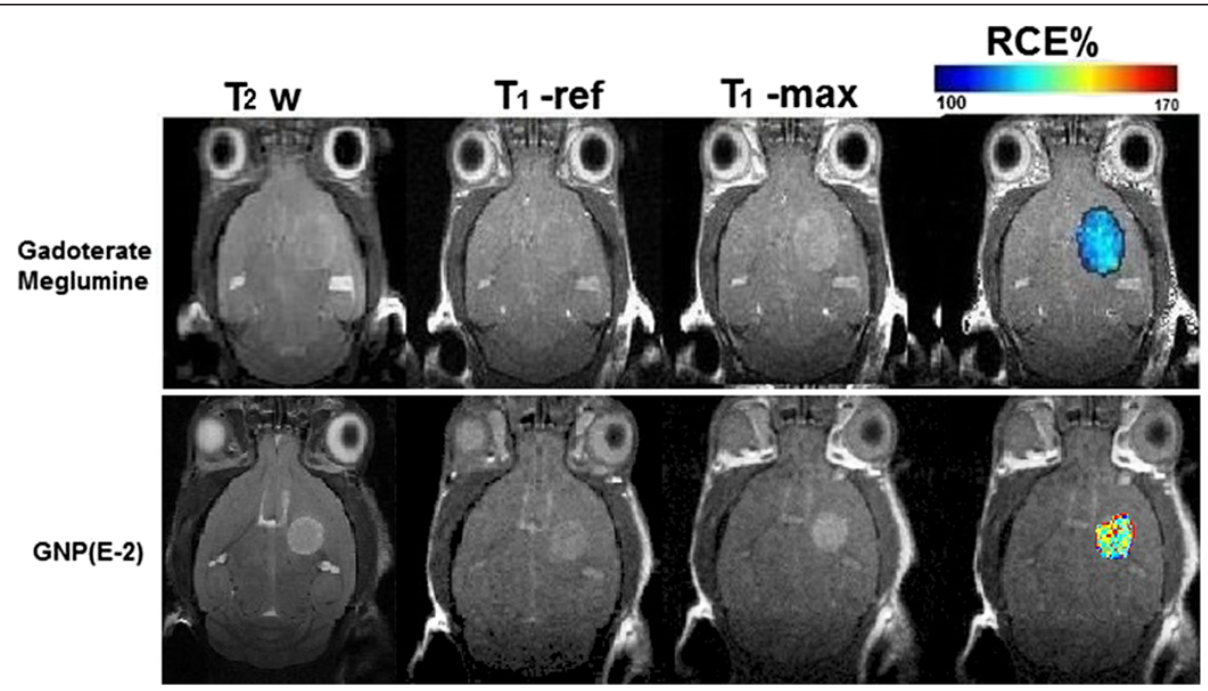

Figure 3 Short name: Coronal $\mathrm{T}_{2}$-weighted images, DCE-MRI images and RCE maps of two mice bearing a GL261 glioma. Detailed legend: From left to right, representative coronal $T_{2}$-weighted images, DCE-MRI images ( $T_{1}$-weighted reference prior to contrast ( $T_{1}$-ref) and at the maximum contrast enhancement point $\left(\mathrm{T}_{1}\right.$-max)), and RCE maps of two mice bearing a GL261 glioma. One animal was studied with gadoterate meglumine (top), and the other with GNP (E_2) (bottom). $T_{1}$-ref images were acquired before injecting the contrast agent bolus while $T_{1}$-max images correspond to the point of maximum contrast enhancement after gadoterate meglumine or GNP administration (see Figure 4). Colour coded bars on top of RCE provide intensity range shown in figures. Black pixels correspond to instances with values above or below the user-established \% RCE postprocessing limits as described in methods. 


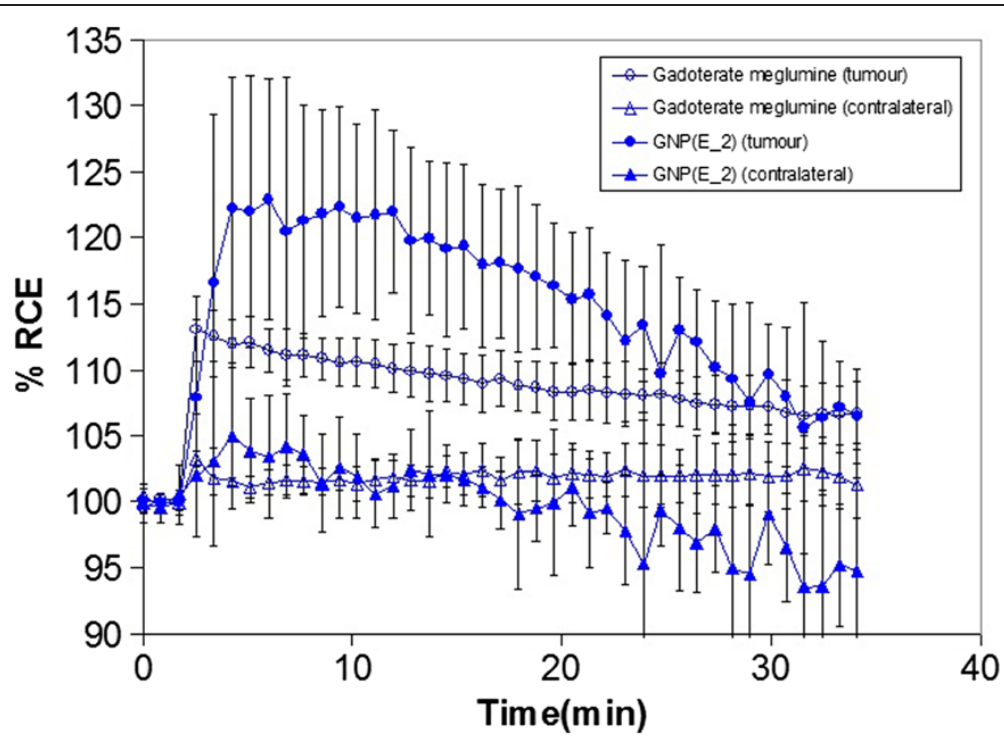

Figure 4 Short name: RCE time-course curves obtained from the quantification of DCE-MRI images. Detailed legend: RCE time-course curves obtained from the quantification of DCE-MRI images. Each curve displays the average contrast enhancement obtained for each group of mice. Values correspond to the results obtained from 3 animals/group and 3 slices/animal, i.e. $n=9$ measurements/group. Gadoterate meglumine, open symbols; GNP (E_2), filled symbols. Tumor is coded by circles and contralateral non-tumoral areas by triangles. Bars show +/-SD.

and Table 1). They were prepared as $\mathrm{T}_{1}$-agents aiming to target mouse brain tumors $[7,31]$ mimicking the multivalent presentation of carbohydrates at the cell surfaces $[32,33]$. These GNPs represent a novel multivalent platform for biological applications [29,34-37], soluble and stable under physiological conditions, and resistant to enzymatic degradation [38]. We selected simple carbohydrates to coat

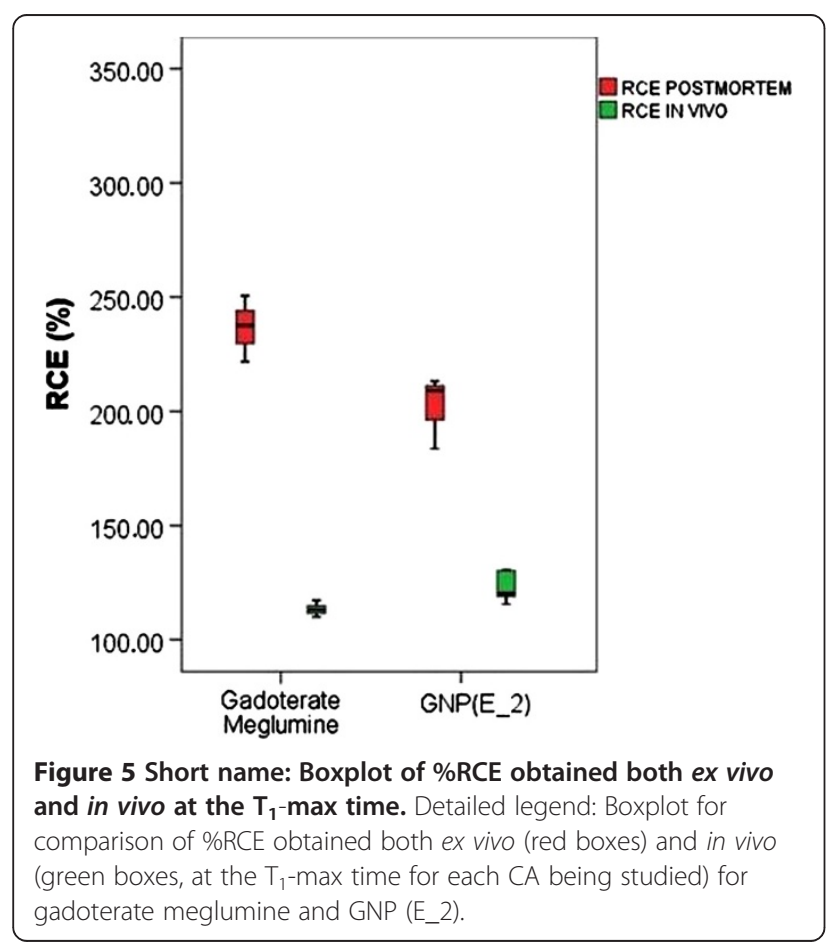

the Gd-based gold nanoparticles, as they are important nutrients for cells. Also, their uptake/binding is dependent on the expression of specific transporters (i.e. GLUTs) [39], and carbohydrate-binding proteins (lectins) which may confer targeting properties to the Gd-GNPs.

Different approaches have been tested to improve the assessment of new CAs, such as: studies with tumor-bearing animals, with no intermediate steps described between the CA evaluation in vitro and in vivo assays [40], computational simulation methods to predict the behaviour of a conditional CA [8], incubation of a CA with a gliosarcoma cell line in well plates to check for interaction and internalization using MRI [41], or incubation of a targeted CA with a cell line over-expressing the folate receptor in order to evaluate the efficiency of the targeting [42]. Those approaches based on in vitro relaxometry measurements incorporating the use of cell lines, are useful for high throughput CA analysis, but do not fully reproduce the in vivo environment [41]. In this respect, the interaction of the $\mathrm{CA}$ with extracellular macromolecules or proteins, may restrict the overall chelate mobility, and thus change its relaxivity [3]. Additionally, post mortem ex vivo approaches have been previously used to approximate the CA performance potential in vivo $[11,12]$. Those ex vivo studies [11,12] were conceptually similar to our herewith described method. Nonetheless, the tissues investigated by them (mouse leg [11] or subconjunctive eye tissue [12]), were not classical tissues for contrast enhancement experiments, as done in our case (brain/brain tumor). Authors in [11] did not proceed into comparing the performance of different CA or validated the possible 


\section{GNP (E_2)}

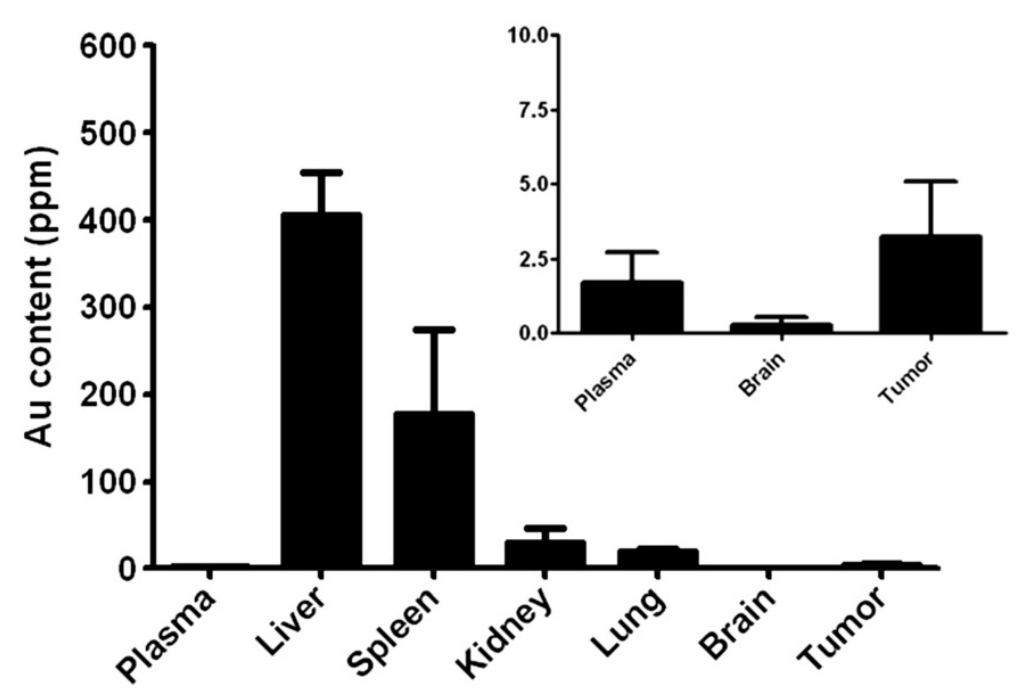

Figure 6 Short name: Gold (Au) biodistribution as measured by ICP-MS 24 h post-administration of GNP (E_2). Detailed legend: Gold (Au) biodistribution as measured by ICP-MS $24 \mathrm{~h}$ post-administration of GNP (E_2). Inset shows tumor Au accumulation at a different scale, compared to element concentrations in plasma and contralateral brain. The difference of Au accumulation between contralateral brain and tumor is nearly significant for GNP (E-2), $p=0.056$. Values are shown as mean parts per million (ppm) and SD (bar). Statistical comparisons correspond to unpaired t-tests.

relevance in an in vivo model. Furthermore, authors in [12] did compare ex vivo post-mortem data with in vivo data, but the emphasis was placed in CA clearance rate changes between low and high molecular weight $\mathrm{CA}$ rather than in the development and evaluation of a method to gauge contrast potential among different agents. Generally speaking, several effects related to the in vivo environment cannot be easily evaluated in vitro. Accordingly, we believe that this biological environment should be mimicked as closely as possible in order to achieve an optimal prediction of the CA potential. In this sense, our method should perform better than previously described strategies $[8,11,12,38-40]$. The results obtained in this study, which will be discussed next, suggest the importance of taking into account not only these CA in vivo environment effects because other factors, such as Gd loading and macromolecular behaviour of the CA, could also play a role and should be considered as relevant.

\section{Effect of nanoparticle coating in positive CA results}

We have previously observed that small modifications in the chemical structure of ligands coating the Gd-GNPs lead to changes in its contrast enhancement effects [7]. This could be due to the Gd hydration number or the overall CA correlation time which, in turn, could affect their relaxivity.

When considering the in vitro relaxivity measurements described in this work, differences (see Table 1) were observed among the GlcCX-DO3AC11 series (GNPs (E_1), (E_2) and F-J, X being a number between 2 and 9). These differences could be influenced by the length of the saccharide's linker, which could modulate the access of water to the $\mathrm{Gd}$ chelated ion. As an example, GlcC3-DO3AC11 (GNP I) has the lowest $r_{1}$ value at $1.4 \mathrm{~T}$ (for glucose GNPs) and tends to be in the low range values region at 7.0 T. On the other hand, there was a tendency to obtain better $r_{1}$ values at $1.4 \mathrm{~T}$ with $\mathrm{C} 5$ linkers (e.g. GNPs (E_2) and J) and the same tendency is observed at 7.0 T, despite a slightly different behaviour is seen in GNP (E_1).

The ex vivo post mortem studies showed discrepant results compared to in vitro $\mathrm{r}_{1}$ values: the GlcC5 linker did not produce a high RCE, as expected from the in vitro $\mathrm{r}_{1}$ results. In this sense, the in vitro high relaxivity values measured for the GNPs (E_2) and (J) did not turn

Table 2 RCE ex vivo, RCE in vivo, $\mathrm{K}_{\mathrm{ep}}$ and IAUC values obtained with in vivo studies

\begin{tabular}{|c|c|c|c|c|c|}
\hline CA & $\%$ Ex vivo $\mathrm{RCE}$ & $\%$ In vivo $\mathrm{RCE}$ at $\mathrm{T}_{1}-\mathrm{max}$ & $\%$ In vivo $\mathrm{RCE}$ at $3 \mathrm{~min}$ & $K_{e p}\left(\min ^{-1}\right)$ & IAUC (A.U.) \\
\hline Gadoterate meglumine & $236.7 \pm 14.4$ & $113.1 \pm 2.5$ & $113.1 \pm 2.5$ & $0.32 \pm 0.36$ & $2.8 \pm 0.1$ \\
\hline GNP (E_2) & $201.9 \pm 16.1$ & $124.9 \pm 8.3^{*}$ & $107.9 \pm 2.0^{*}$ & $0.06 \pm 0.03^{*}$ & $2.6 \pm 0.2^{*}$ \\
\hline
\end{tabular}

Detailed legend:

\% RCE in vivo is shown both at $\mathrm{T}_{1}$-max of each $\mathrm{CA}$ and at $3 \mathrm{~min}$ after their administration.

${ }^{*} \mathrm{p}<0.05$ in comparison with gadoterate meglumine. 


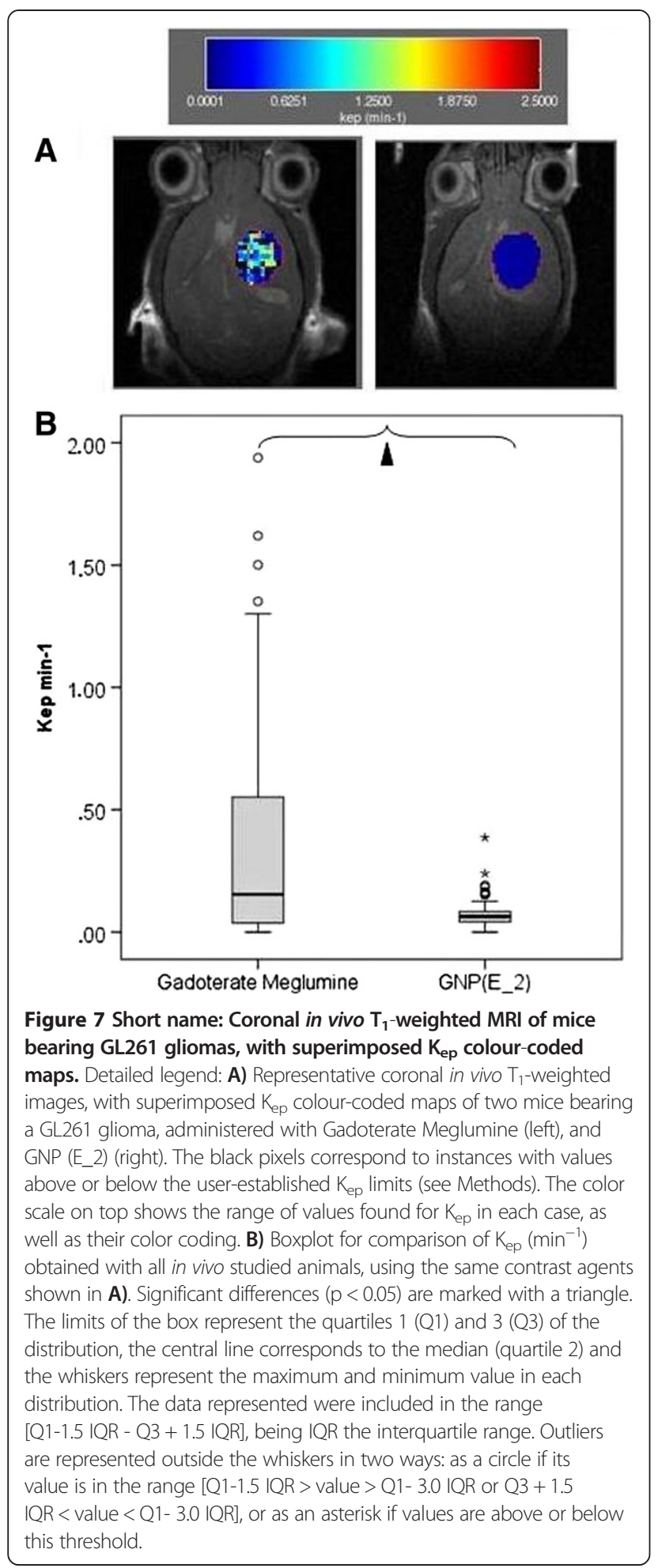

into high contrast enhancement ex vivo. It is also feasible that the Gd loading (between 3.2 and 7\% Gd in weight of nanoparticle, see Table 1 for details) of these GNPs, could have an influence in the RCE obtained in each case, even if correcting the preparation of the CAs solutions to achieve the same final concentration of Gd for ex vivo post mortem studies. In this respect, the percentage $\mathrm{Gd}$ content per nanoparticle significantly correlates with $r_{1}$, but not $r_{2}$ (Additional file 1: Figure S1). Thus the beneficial effect of high payload per nanoparticle has been demonstrated for positive contrast targeted nanoparticles in ex vivo studies with cultured cells [43]. This fact could also explain the differences between GNPs (E_1) and (E_2), which have the same structural formula but different percentage of Gd (per mass of GNP), and different $r_{1}$ values in vitro as well as different RCE ex vivo. Moreover, the nature of the carbohydrate may play a role in the ex vivo post-mortem RCE. This could be observed for GNP $(J)$, coated with galactose, where the high $r_{1}$ values at 1.4 $\mathrm{T}$ and 7.0 T did not turn into high RCE ex vivo. It is also worth pointing out the possible $r_{2}$ contribution which could counteract the $\mathrm{r}_{1}$ effect [44], and, therefore, the overall RCE (e.g. GNP (E_1), $\mathrm{r}_{2} / \mathrm{r}_{1}=7.48$ and \%RCE $=$ 182.8; GNP (E_2), $\mathrm{r}_{2} / \mathrm{r}_{1}=1.77$ and $\% \mathrm{RCE}=201.9$, see Table 1 for further details).

Additionally, the length of the alkane linkers for glucose may modulate the interactions with the cellular receptors, and, accordingly the targeting of the investigated nanoparticles. It is well known that GLUT-1 transporters for glucose are overexpressed in tumor cells [45] and its structure has been extensively investigated. In this respect, an "optimal" length of the saccharyde linker in the CA glucose series may be required to properly interact with the GLUT-1 transporters, which would translate into maximum restriction of the $\mathrm{Gd}^{3+}$ chelate mobility and low correlation times. The length of the alkane linker may modulate these interactions. In this sense, the "C3-linker" (GNP I) produced one of the lowest ex vivo RCE values measured in the GlcCX-DOTAC11 series. We also found significant differences compared to $\mathrm{C} 5$ and $\mathrm{C} 9$ linkers (GNP (E_1) and GNP (G)). This would suggest that a glucose linker arm too short could compromise proper interaction of the GNP with GLUT transporters, although additional studies will be needed to fully evaluate this possibility. Furthermore, results in [46] provide some support to the "targeting" hypothesis by showing that glucose-coated niosomes produced contrast-enhancement, whereas niosomes without glucose produced no enhancement, reinforcing the possibility that this interaction nanoparticletransporter is also present in our case. Besides to possibly modulating ex vivo/in vivo CA relaxivity values, the use of glucose could also help targeting the CA-containing nanoparticles to tumor cells by means of their glucose transporters.

Preliminary results comparing the in vivo effects of a similar GNP (with the same structural formula as GNP E_1 and E_2) in the same tumor model did not show significant differences in the maximum RCE changes observed during different glycemic states, euglycemia 
and hyperglicemia $(26.9 \pm 1.8 \mathrm{mM}$ in blood) (results not shown). This suggests that the possible competition between the glucose GNPs and free glucose for the interaction with GLUT transporters does not seem to affect their performance as $T_{1}$ contrast agents. Nonetheless, further assays on the in vivo competition between glucose GNPs with and without chelated Gd should help to clarify the point about the contribution of the glucose GNP binding to GLUTs in the measured RCE.

The in vitro $\mathrm{r}_{1}$ relaxivity value for GNP (E_2) at $7 \mathrm{~T}$ was the best among the Gd-GNPs (Table 1), but the ex vivo analysis did suggest slightly, but significant, worse performance than gadoterate meglumine. The ex vivo and in vivo RCE measurements both predicted worse performance of GNP (E_2) compared to gadoterate meglumine when RCE was measured at the same time point (3 min post CA injection, time of maximum contrast enhancement for gadoterate meglumine) (Table 2). However, when RCE was measured at the time of maximum enhancement for each CA in the DCE-MRI experiments, the GNP (E_2) performed significantly better than gadoterate meglumine (see Table 2 and Figure 5). Significant differences were found in the maximum time of contrast enhancement (" $\mathrm{T}_{1}$-max") between the two analyzed CA, and this points to the fact that the behaviour of GNP (E_2) is the expected one for a macromolecular-like contrast agent [12]. This may be due to the well known "enhanced retention" (EPR) effect of nanoparticulated CAs in tumors [47]. The described diameter of the GNPs is between 1.8-4.5 nm [7], comparable to agents used as macromolecular contrast agents $(4-6 \mathrm{~nm},[12,48])$, and therefore reach the time to maximum enhancement more slowly than free gadolinium chelate (gadoterate meglumine) (Figure 4). This result agrees with previous results obtained using niosomes by authors in [46] although with a different tumor type. Finally, the gold biodistribution found in our study (e.g. maximal accumulation found in liver and spleen, Figure 6) is in good agreement with literature for gold nanoparticles $[49,50]$.

The pharmacological parameters measured for gadoterate meglumine and GNP (E_2) (e.g. $K_{\text {ep }}$, see Figure 7 and Table 2) were significantly different. Authors in [12] have previously shown that CAs with different molecular sizes also have different clearance rates (lower $\mathrm{K}_{\mathrm{ep}}$ values), which becomes slower with higher molecular weight CAs. Authors in [48] have measured a closely related parameter, the "transfer coefficient" $\left(\mathrm{K}_{\mathrm{PS}}\right)$ (transfer rate of the contrast agent from the blood to the interstitial space, highly dependent on the permeability), and found an inverse relationship between the molecular weight of the CA and the transfer coefficient. Then, GNP (E_2) behaves as a macromolecular contrast agent, according to their average $\mathrm{K}_{\mathrm{ep}}$ values.
Finally, in addition to the more accurate simulation of the in vivo conditions, the ex vivo post mortem method described here consumes a very small amount of CA compared to an in vivo study: 800 times less in the case of positive contrast agents $(5 \mathrm{nmol} /$ animal vs. $4,000 \mathrm{nmol} / \mathrm{animal}$ ) and it is applicable both to positive and negative (Supplementary Material and [51]) CA. Furthermore, as it is carried out with sacrificed animals, no preliminary toxicological assays are required. It is worth pointing out that, although this method is better at simulating the in vivo conditions, the use of animals is costly and it would require certain facilities and compliance of legal/ethical aspects, depending on the country were researchers are based. The development of new and improved in vitro strategies, able to evaluate cells exposing groups or proteins able to be targeted, could also offer an intermediate and useful step in this field.

\section{Conclusions}

In summary, we have developed a low-consumption ex vivo method for testing the potential in vivo performance of new contrast agents. This should allow us to choose from a series of new candidate CAs the one/s more likely to perform better in vivo. This method requires small amounts of CA (e.g. $5 \mathrm{nmol} /$ animal) and should allow a more rationally informed selection, avoiding unnecessary in vivo and toxicology tests for the ex vivo poorly performing substances. This reduces animal needs, material and overall costs. Nevertheless, caution should be taken when evaluating CAs with different kinetic behaviour (e.g. small vs. high molecular weight $\mathrm{CAs}$ ). In this case, the ex vivo method could produce an underestimation of the actual contrast enhancement potential. Further studies may be needed in order to fully optimize the currently described protocol.

\section{Methods}

\section{Synthesis of contrast agents}

\section{Gd-complexes containing glyconanoparticles (Gd-GNP)}

The positive contrast agents (Gd-GNPs) evaluated in this work were 1.8-4.5 nm sized gold nanoparticles coated with multiple copies of sugar conjugates and 1, 4, 7, 10-tetraazacyclododecane-1, 4, 7-triacetic acid (DO3A) Gd-complexes. The basic structures and gadolinium content of each Gd-GNP type are schematized in Figure 1 and listed in Table 1. The Gd-GNPs were prepared by ligand place exchange reaction (LPE) starting from glyconanoparticles (GNPs) coated only with sugars as described in [13,31] (Gd-GNPs E_1, E_2 and F-J). The sugars (glucose, galactose) are conjugated to an aliphatic chain of variable number of carbon atoms $(\mathrm{C} 2, \mathrm{C} 3, \mathrm{C} 5$, C7, C9, C11), while the DO3A derivatives contain a C11 aliphatic chain (Table 1). The obtained Gd-GNPs were characterized by TEM, UV-vis, IR, ${ }^{17} \mathrm{O}$ NMR 
and ICP-AES as described in [7] and [31]. The average diameter of the gold core was 1.5-2.5 nm and the number of sugars and Gd chelates on the surface ranged between 70-130 molecules for sugars and 14-34 for Gd chelates. The amount of Gd (III) was between $3.2 \%$ and $7.0 \%$, determined by ICP-AES (induced coupling plasma-absorption spectroscopy).

\section{In vitro relaxivity studies \\ Studies at $1.4 T$}

The longitudinal $\mathrm{T}_{1}$ relaxation times of Gd-GNPs were determined at $10,5,2.5,1.25,0.625 \mathrm{mg} \mathrm{mL}^{-1}$ concentrations of Gd-GNPs in water (Gd concentration ranged from 0 to $3 \mathrm{mM})$ at $60 \mathrm{MHz}(1.41 \mathrm{~T})$ and $37^{\circ} \mathrm{C}$ in a Bruker Minispec MQ-60 NMR spectrometer (Bruker Optik, Ettlingen, Germany) at CICbiomaGUNE, San Sebastián, Spain. The values of $T_{1}$ were determined using the inversion-recovery method. The calculation of the $T_{1}$ was carried out using monoexponential fitting. Three independent measurements of $\mathrm{T}_{1}$ were performed in every sample for statistical analysis purposes.

Relaxivities $r_{1}$ and $r_{2}$ were obtained from the slopes of the curves $1 / T_{1}$ and $1 / T_{2}$ vs the concentration of $\mathrm{Gd}$ expressed in $\mathrm{mM}$.

\section{Studies at 7.0 T}

Four different solutions ranging from 0.125 to $1 \mathrm{mM}$ in gadolinium of Gd-GNPs and gadoterate meglumine in saline solution $(\mathrm{NaCl} 0.9 \%$, B.Braun) were used. A phantom using capillary tubes (1.5 $\mathrm{mm}$ internal diameter) for GNP solutions, immobilized in plasticine was used for MR scanning.

All $7 \mathrm{~T}$ studies (in vitro relaxivity, ex vivo post mortem and in vivo) were carried out at the joint NMR facility of the Universitat Autònoma de Barcelona and CIBER-BBN (Cerdanyola del Vallès, Spain), using a $7 \mathrm{~T}$ horizontal magnet (BioSpec 70/30; Bruker BioSpin, Ettlingen, Germany) equipped with actively shielded gradients (B-GA12 gradient coil inserted into a B-GA20S gradient system). A quadrature $72 \mathrm{~mm}$ inner diameter volume coil was used for in vitro studies whereas a quadrature receive surface coil, actively decoupled from a linear volume resonator with $72 \mathrm{~mm}$ inner diameter was used for in vivo and ex vivo studies. The in vivo studies were performed at $37^{\circ} \mathrm{C}$, whereas the in vitro relaxivity and ex vivo post mortem studies were performed at $23^{\circ} \mathrm{C}$. A recirculating water-system, incorporated to the animal bed, was used to control the temperature, measured by a probe and was constantly monitored (SA Instruments, Inc., New York, USA).

All studies started with acquiring fast $\mathrm{T}_{2}$-weighted images in three orthogonal planes using a Rapid Acquisition by Relaxation Enhancement (RARE) sequence; Rare Factor, 8; field of view (FOV), 19.2×19.2 mm; matrix size (MTX), $128 \times 128(150 \times 150 \mu \mathrm{m} /$ pixel); number of slices
(NS), 14 (coronal), 7 (sagittal) and 5 (axial); slice thickness (ST), $1 \mathrm{~mm}$; interslice-thickness (IST), $1.1 \mathrm{~mm}$; TR/TE, 2000/36 ms; number of averages (NA), 1; total acquisition time (TAT), $24 \mathrm{sec}$.

After these initial sequences, relaxivity studies $\left(T_{1}\right.$ and $\mathrm{T}_{2}$ maps) for Gd-GNPs were carried with the following parameters:

For $\mathrm{T}_{1}$ maps acquisition, a RARE-VTR sequence was chosen with RARE factor $=4$; FOV, $7.0 \times 3.5 \mathrm{~cm}$; MTX, $512 \times 256(137 \times 137 \mu \mathrm{m} /$ pixel $) ; \mathrm{NS}, 1$; ST, $1 \mathrm{~mm}$; TE $\mathrm{eff}_{\text {, }}$ $30 \mathrm{~ms}$; TRs were according to the following list: 75, 150, 300, 600, 1200, 2400 and $4800 \mathrm{~ms}$; NA, 2; TAT, 20 min. For $\mathrm{T}_{2}$ maps acquisition, a Multi-Slice-Multi-Echo (MSME) sequence was used; FOV, $7.0 \times 3.5 \mathrm{~cm}$; MTX, $512 \times 256(137 \times 137 \mu \mathrm{m} /$ pixel $) ; \mathrm{NS}, 1$; ST, $1 \mathrm{~mm}$; TR, $4800 \mathrm{~ms}$; TEs were according to the following list: 20 , 40, 60, 80, 100, 120, 140, 160, 180, 200, 220, 240, 260, 280, 300, 320, 340, 360, 380 and $400 \mathrm{~ms}$; NA, 4; TAT, $1 \mathrm{~h} 16 \mathrm{~min}$.

\section{Animal models}

The animal model used for these studies was C57BL/6 healthy female control mice of 20-25 g weight. All C57BL/6 animals were obtained from Charles River Labs (France) and housed at the Animal Facility of the Universitat Autònoma of Barcelona.

For the ex vivo post mortem studies $\mathrm{n}=8$ (one for each $\mathrm{CA}$ ) were used for the CA characterization procedures. For the in vivo study, $\mathrm{n}=6$ mice harbouring a GL261 glioblastoma were used. For the orthotopic model generation, the GL261 mouse glioma cell line was obtained from the Tumour Bank Repository at the National Cancer Institute, Frederick, MD, USA. Cells were grown in RPMI-1640 culture medium supplemented with $2.0 \mathrm{~g} / \mathrm{L}$ sodium bicarbonate, $0.285 \mathrm{~g} / \mathrm{L} \mathrm{L}$-glutamine, $10 \%$ foetal bovine serum and $1 \%$ penicillin-streptomycin solution. Culture medium and chemicals were purchased from Sigma-Aldrich (St. Louis, MO, USA) unless otherwise indicated. Culture plastic was obtained from Nunc (Roskilde, Denmark).

Tumors were induced in the six mice by intracranial stereotactic injection of GL261 cells into the caudate nucleus, essentially as described in [52]. MRI studies were performed approximately 2 weeks after cell inoculation.

All studies were carried out according to protocols approved by the local/institutional ethics committee, according to the regional and state legislation (CEEAH 1178 and 1176).

\section{Ex vivo and in vivo MRI studies}

These studies were carried out at the joint NMR facility of the Universitat Autònoma de Barcelona and CIBER-BBN (Cerdanyola del Vallès, Spain), using a $7 \mathrm{~T}$ horizontal magnet. 
All studies started with high resolution $\mathrm{T}_{2}$-weighted images which were acquired from three horizontal sections of the brain in order to have a good morphological characterization of the investigated tissue. The sequence used for this purpose was RARE with the following parameters: RARE factor $=8$, FOV, $17.6 \times 17.6 \mathrm{~mm}$; MTX, 256×256 $(69 \times 69 \mu \mathrm{m} /$ pixel); ST, $1 \mathrm{~mm}$; NS, 3; TR/TE, 3000/36 ms; NA, 4; TAT, 4 min $48 \mathrm{sec}$.

\section{Ex vivo post mortem studies}

The CAs to be used for post mortem ex vivo evaluation were dissolved in saline solution $(0.9 \% \mathrm{NaCl}, \mathrm{B}$. Braun $)$ taking into account the estimation of Gd content previously determined (see Table 1), in order to achieve solutions of comparable concentrations of the desired metal. The amount finally used for each animal was $5 \mathrm{nmol}$ of $\mathrm{Gd}$ dissolved in $4 \mu \mathrm{l}$ of saline solution. A commercial solution of Gd CA (Gadoterate Meglumine, DOTAREM ${ }^{\circledR}$, Guerbet, Roissy, France) was used as a standard to compare the enhancement obtained by MRI with the GNPs.

A $\mathrm{T}_{1}$-weighted image acquisition was performed using the same coronal sections described previously in "in vitro 7 T studies". For this, a MSME sequence was used with: FOV $17.6 \times 17.6 \mathrm{~mm}$; MTX, $128 \times 128$ matrix $(138 \times 138 \mu \mathrm{m} /$ pixel); TR/TE, 350/8.5 ms; NA, 1; number of repetitions (NR), 15; TAT, $11 \mathrm{~min}$. Animals were anesthetized and handled as described for "in vivo studies". After that, animals were sacrificed with an overdose of intraperitoneal sodium pentobarbital (200 mg/kg) (Vetoquinol, Madrid, Spain), and then immobilized on a stereotactic holder (Kopf Instruments, Tujunga, CA, USA). The contrast administration was carried out as described for tumor generation [52], except that the cell suspension was replaced with the contrast agent solution to be evaluated, and 3 injection points at each side were administered, as follows: $1.0 \mathrm{~mm}$ holes were made lateral (right) to the midline, $2.32 / 2.32 / 2.00 \mathrm{~mm}$, and with the following " $\mathrm{Y}$ " coordinates along the midline: $0.10 / 1.5 / 4.5 \mathrm{~mm}$. The whole process of CA injection ex vivo took 15 minutes. After this, the $T_{1}$ weighted image acquisition was repeated as above.

\section{In vivo studies}

Anesthesia was performed with isoflurane (B.Braun, Melsungen, Germany) at $0.5-1.5 \%$ in $\mathrm{O}_{2}$, maintaining the respiratory frequency between $40-60$ breaths $/ \mathrm{min}$. Body temperature was maintained between $36.5-37.5^{\circ} \mathrm{C}$ with a recirculating water system incorporated in the animal bed, and measured with a rectal probe. Respiration rate and temperature were constantly monitored (SA Instruments, Inc., New York, USA). Before immobilization in the animal holder, each mouse was cannulated in the tail vein using a home-built multi-delivery polyethylene tubing system. In this case, a 30G 2-way catheter was connected, through polyethylene tubing, to 2 independent $1 \mathrm{~mL}$ syringes (Becton-Dickinson S.A., Madrid, Spain) loaded with heparinized-saline $(40 \mathrm{U} / \mathrm{ml})(0.9 \% \mathrm{NaCl}$, B.Braun and heparin, Mayne Pharma España, Madrid, Spain) and one contrast agent $(9.7 \mathrm{mM}$ in $\mathrm{Gd})$. The contrast agent was injected into the mice as a bolus $(72-84 \mu \mathrm{L}$, about $0.04 \mathrm{mmol} / \mathrm{kg}$ ) during Dynamic Contrast Enhanced (DCE)- $\mathrm{T}_{1}$ MRI studies. Three glioma-bearing mice were injected with gadoterate meglumine and another three were injected with GNP (E_2) (see Results).

A DCE $\mathrm{T}_{1}$ study was then performed using three coronal sections. For this, a MSME sequence was used with: MTX, $128 \times 128$ matrix $(138 \times 138 \mu \mathrm{m} /$ pixel $)$; TR/TE, $200 / 8.5 \mathrm{~ms}$; ST, $1 \mathrm{~mm}$; NA, 2; NR, 41; TAT, $35 \mathrm{~min}$. The contrast bolus was administered after the third repetition of the complete $\mathrm{T}_{1}$-weighted sequence (about $2.5 \mathrm{~min}$ after the start of the image acquisition protocol).

\section{Biodistribution of Gd-GNP by ICP-MS}

At established time-points (24 h, 48 h, see further details in the Additional file 1: Table S1) after GNP or vehicle administration, animals ( $\mathrm{n}=3$ for each time point) were euthanized by cervical dislocation and required tissues (liver, kidney, spleen, brain and tumor among others) as well as urine and blood samples were collected and stored at $-80^{\circ} \mathrm{C}$. Samples were analyzed by Induced Coupled Plasma-Mass Spectrometry (ICP-MS) at the Unidad de Análisis Elemental of the Scientific Technical Facilities of the University of Barcelona.

\section{Histopathological analysis}

At established time-points (24 h, $48 \mathrm{~h}$ and 14 days) after GNP or vehicle administration administration, animals ( $n=3-6$ for each time point) were sacrificed by cervical dislocation. The required tissues were dissected, fixed and stained with Haematoxilin-Eosin.

\section{Processing and post-processing of MR data}

Ex vivo post mortem studies: all processing and post-processing of $\mathrm{T}_{1}$-weighted images were carried out with Bruker software Paravision (version 4.0) and ImageJ 1.44p (National Institutes of Health, USA ${ }^{\mathrm{a}}$ ). Three regions of interest (ROIs), corresponding to the coordinates described in the "ex vivo post-mortem studies" section were manually defined after visual inspection both in the area of maximum enhancement and equivalent area of contralateral parenchyma. The relative contrast enhancement (RCE) - injection site ROI vs. contralateral parenchyma - obtained in each case was used for calculations (see Equation 1). Only the slice with better defined contrast-enhanced region was measured.

$$
R C E(\%)_{e x_{\text {vivo }}}=\left(\frac{S(i)}{S(c)}\right) \times 100
$$


Where S (i) is the absolute signal intensity of the "ipsilateral" region with respect to the contrast administration and that visually shows contrast enhancement, and $\mathrm{S}$ (c) is the absolute signal intensity of the equivalent contralateral region, which serves as a control and that is defined as "100\%".

In vivo studies: DCE-MRI data were processed using a platform for pharmacokinetic analysis recently developed within the intramural CIBER-BBN project PROGLIO/ PROGLIO2 using IDL (ITT Visual Information Solutions, Boulder, CO, USA) available for download at http://www. die.upm.es/im/archives/DCEurLAB/ from the Biomedical Images Technologies research group of the Universidad Politécnica de Madrid (BIT-UPM) [53], and also with additional home written scripts in IDL as described in [54]. RCE in vivo was calculated for each pixel as the ratio between the maximum signal enhancement and the average intensity before bolus injection unless otherwise indicated, and the three slices acquired were used for this calculation. In this way, time-course curves that quantified the average contrast enhancement inside the tumors and in surrounding non tumor-bearing areas of the brain parenchyma (defined from ROIs) were generated. Moreover, color-coded maps that translated the maximum contrast enhancement at each pixel of the FOV at the time of maximum enhancement were also generated (RCE). Maps with the initial area under the curve (IAUC) were generated by integration of the dynamic curves during the first 150 seconds after contrast agent administration, as in $[55,56]$ but without normalization to a reference IAUC value - a constant arterial input function was assumed. $\mathrm{K}_{\text {ep }}$ parameters (Eq. 2) were calculated according to the "Hoffmann-Brix" model [57]:

$$
\frac{S(t)}{S(0)}=1+A k_{e p} \frac{\left(e^{-k_{e p} t}-e^{-k_{e l} t}\right)}{k_{e p}-k_{e l}}
$$

being $K_{e l}$ the elimination rate of the contrast agent by the plasma. Thus, the initial slope of the kinetic curve, detected at short times after injection, is proportional to A.K $\mathrm{K}_{\mathrm{ep}}$, being A the initial upslope of the enhancement curve.

For each animal and slice, ROIs were manually drawn around the tumor. The resolution of all datasets was reduced from $128 \times 128$ to $64 \times 64$ pixels and RCE, IAUC, and $K_{\text {ep }}$ maps were generated within those ROIs. The maps were coloured according to a scale comprising the maximum and minimum values manually defined. Pixels with values above or below these limits were coloured black. The whole adjustment of each pixel (including fitting deviation and mean errors) could be exported in an excel format. This helped improving the accuracy of the "Hoffmann-Brix" model, allowing us to detect inadequate fittings which were filtered-out by defining thresholds for standard deviation $(<0.5)$ and mean error $(<1.5)$, above which pixels were discarded from the analysis. Statistical analysis within the tumor regions on each slice were carried out with SPSS 19.0 (SPSS Inc., Chicago, USA). A total of 836 and 699 pixels were analyzed from animals studied with gadoterate meglumine and GNP (E_2) injection, respectively.

\section{Statistical analysis}

Ex vivo post mortem studies: the overall signal change measured in $\mathrm{T}_{1}$ weighted MRI ROIs for each GNP was compared with gadoterate meglumine $[7,58]$ and saline solution.

In vivo studies: measurements from the same animal but corresponding to different slices were considered as independent measurements/cases (total $n=9$ for each CA). For in vivo studies, the average contrast enhancement in the ROI inside the tumors was also compared among animals injected with different contrast agents.

For both studies, normality was first inspected in each group by the Kolmogorov-Smirnov test and variance homogeneity with the Levene test. A two-tailed Student's $\mathrm{t}$-test for independent measurements was used for statistical analysis when data followed a normal Gaussian distribution. If data had a non-normal distribution, Mann-Whitney $U$ test was used for statistical analysis. The significance level for all tests was $\mathrm{p}<0.05$.

\section{Endnotes}

${ }^{a}$ http://rsb.info.nih.gov/ij/.

\section{Additional file}

Additional file 1: 1. Biodistribution and toxicological studies carried out with GNP E_1 and E_2. 2. Application of the ex vivo postmortem method to iron oxide nanoparticles.

\section{Abbreviations}

A.K $\mathrm{K}_{\text {ep }}$ : Amplitude times exchange rate constant $\mathrm{K}_{\text {ep; }}$ BBB: Blood brain barrier; CA: Contrast agent; DCE: Dynamic contrast enhancement;

DMSA: Dimercaptosuccinic acid; FOV: Field of view; GBM: Glioblastoma; Gd-GNP: Gadolinium-based glyconanoparticle; GM: Gadoterate meglumine; IAUC: Initial area under the curve; ICP-AES: Induced coupling plasma-atomic emission spectroscopy; IDL: Interface description language; IR: Infrared spectroscopy; IST: Inter-slice distance; Kel: Elimination rate constant by the plasma; $K_{\text {ep }}$ : Efflux rate constant from extravascular extracellular space to plasma; MRI: Magnetic resonance imaging; MSME: Multi-slice-multi-echo; MTX: Matrix size; NA: Number of averages; NEX: Number of excitations; NR: Number of repetitions; NS: Number of slices; PBS: Phosphate-buffered saline solution; RARE: Rapid acquisition by relaxation enhancement; RCE: Relative contrast enhancement; ROI: Region of interest; SI: Signal intensity; SPION: Superparamagnetic iron oxide nanoparticles; ST: Slice

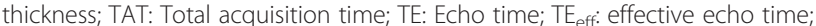
TEG: Triethylene glycol; TEM: Transmission electron microscopy; TR: Recycling time; UV-vis: Ultraviolet-visible spectroscopy. 


\section{Authors' contributions}

MA, RVS and TDG performed the acquisition of ex vivo and in vivo MRI data, as well as relaxivity data at 7 T. SLP set up the 7 T MRI/MRS sequences. Al and MM carried out the glyconanoparticle synthesis, evaluation and relaxivity studies at 1.4 T. SP was responsible for design and supervision of glyconanoparticle synthesis and characterization. IA and SSJr performed the biodistribution studies. NMS and OBM participated in SPION synthesis and characterization, as well as relaxivity studies at 1.4 T. JS was responsible for design and supervision of SPION synthesis and characterization. CA was responsible for the design of the experimental part of the study and also in the analysis and interpretation of critical data. APC participated in the supervision of preclinical studies and acquisition of MRI in vivo data, being also responsible for the first draft of the manuscript. All authors participated in the revision and approval of the manuscript final version.

\section{Acknowledgements}

Work funded by MICINN or MINECO grants PHENOIMA (SAF 2008-0332), MARESCAN (SAF 2011-23870), NANBIOCEL (CTQ2008-04638) and MULTIGLYCONANO (CTQ2011-27268); Centro de Investigación Biomédica en Red - Bioingeniería, Biomateriales y Nanomedicina (CIBER-BBN, [http://www.ciber-bbn.es/en]), an initiative of the Instituto de Salud Carlos III (Spain) co-funded by EU FEDER funds; IMAFEN (2008-2009) PROGLIO (2010-2011) and PROGLIO2 (2012-2013), intramural projects of CIBER-BBN. OBM thanks the financial support from the "Ramón y Cajal" MINECO Program. Authors would like to thank Andrés Santos, Juan Ortuño and María Ledesma from Universidad Politécnica de Madrid, for providing access to the IDL-based DCE-MRI data processing platform and for help with its use. Authors would also like to thank Guerbet, for providing free of charge access to Sinerem (Ferumoxtran-10) as control sample.

\section{Author details}

${ }^{1}$ Centro de Investigación Biomédica en Red - Bioingeniería, Biomateriales y Nanomedicina (CIBER-BBN), Spain. ²Departament de Bioquímica i Biologia Molecular, Unitat de Bioquímica de Biociències, Edifici Cs, Universitat Autònoma de Barcelona, Cerdanyola del Vallès, Barcelona 08193 Spain. ${ }^{3}$ Institut de Biotecnologia i de Biomedicina, Universitat Autònoma de Barcelona, Cerdanyola del Vallès, Barcelona 08193 Spain. ${ }^{4}$ Servei de RMN, Universitat Autònoma de Barcelona, Edifici C, Cerdanyola del Vallès, Barcelona 08193 Spain. ${ }^{5}$ Centro de Investigación Cooperativa en Biomateriales - CIC biomaGune, Pa Miramón182, San Sebastián 20009 Spain. ${ }^{6}$ Departamento de Física Aplicada, Facultad de Ciencias, Universidad Autónoma de Madrid, Cantoblanco, Madrid 28049 Spain. ${ }^{7}$ Instituto de Investigación en Nanociencia de Aragón (INA), Edificio Interfacultades II. C/ Pedro Cerbuna, 12. Universidad de Zaragoza, Zaragoza 50009 Spain. ${ }^{8} \mathrm{CIBBIM}$-Nanomedicine, Vall d'Hebron Institut de Recerca (VHIR), Hospital Universitari Vall d'Hebron, Universitat Autònoma de Barcelona, Barcelona 08035 Spain.

Received: 13 December 2013 Accepted: 18 March 2014 Published: 5 April 2014

\section{References}

1. Caravan P, Ellison JJ, McMurri TJ, Lauffer RB: Gadolinium (III) chelates as MRI contrast agents: structure, dynamics, and applications. Chem Rev 1999, 99:2293-2352.

2. Corot $C$, Robert $P$, Idée JM, Port M: Recent advances in iron oxide nanocrystal technology for medical imaging. Adv Drug Deliv Rev 2006, 58:1471-1504

3. Caravan P: Protein-targeted gadolinium-based Magnetic Resonance Imaging (MRI) contrast agents: design and mechanism of action. Acc Chem Res 2009, 42:851-862.

4. Caravan P: Strategies for increasing the sensitivity of gadolinium based MRI contrast Agents. Chem Soc Rev 2006, 35:512-523.

5. Mustafi D, Peng B, Foxley S, Makinen MW, Karcmar GS, Zamora M, Ejnik J, Martin $\mathrm{H}$ : New vanadium-based magnetic resonance imaging probes: clinical potential for early detection of cancer. J Biol Inorg Chem 2009, 14:1187-1197.

6. Battistini E, Gianolio E, Gref R, Couvreur P, Fuzzerova S, Othman M, Aime S, Badet $B$, Durand $P$ : High-relaxivity magnetic resonance imaging (MRI) contrast agent based on supramolecular assembly between a gadolinium chelate, a modified dextran, and poly-beta-cyclodextrin. Chemistry 2008, 14:4551-4561.
7. Marradi M, Alcántara D, de la Fuente J, García-Martín M, Cerdan S, Penadés S: Paramagnetic Gd-based gold glyconanoparticles as probes for MRI: tuning relaxivities with sugars. Chem Commun 2009, 26:3922-3924.

8. Cosentino U, Pitea D, Moro G, Saracino GAA, Villa A: Conformational behaviour determines the low-relaxivity state of a conditional MRI contrast agent. Phys Chem Chem Phys 2009, 11:3943-3950.

9. Gheorghe DE, Cui L, Karmonik C, Brazdeikis A, Penaloza JM, Young JK, Drezek RA, Bikram M: Gold-silver alloy nanoshells: a new candidate for nanotherapeutics and diagnostics. Nanoscale Res Lett 2011, 6:554.

10. Krause W: Contrast Agents I: Magnetic Resonance. Berlin: Springer; 2002.

11. Bouchard L-S, Anwar MS, Liu GL, Hann B, Gray JW, Wang X, Pines A, Chen FF: Picomolar sensitivity MRI and photoacoustic imaging of cobalt nanoparticles. Proc Natl Acad Sci U S A 2009, 106:4085-4089.

12. Kim SH, Csaky KG, Wang NS, Lutz RJ: Drug elimination kinetics following subconjunctival injection using dynamic contrast-enhanced magnetic resonance imaging. Pharmacol Res 2008, 25:512-520.

13. Irure Yoldi A, Marradi M, Arnáiz B, Genicio N, Padroc D, Penadés S: Sugar/gadolinium-loaded gold nanoparticles for labelling and imaging cells by magnetic resonance imaging. Biomater Sci 2013, 1:658-666.

14. Miguel-Sancho N, Bomatí-Miguel O, Colom G, Salvador J-P, Marco M-P, Santamaría J: Development of stable, water-dispersible, and biofunctionalizable superparamagnetic iron oxide nanoparticles. Chem Mater 2011, 23:2795-2802.

15. Küstermann E, Himmelreich U, Kandal K, Geelen T, Ketkar A, Wiedermann D, Strecker C, Esser JSA, Hoehn M: Efficient stem cell labeling for MRI studies. Contrast Media Mol Imaging 2008, 3:27-37.

16. Figuerola A, Di Corato R, Manna L, Pellegrino T: From iron oxide nanoparticles towards advanced iron-based inorganic materials designed for biomedical applications. Pharmacol Res 2010, 62:126-143.

17. Yu MK, Park J, Jon S: Targeting strategies for multifunctional nanoparticles in cancer imaging and therapy. Theranostics 2012, 2:3-44.

18. Gupta AK, Gupta M: Synthesis and surface engineering of iron oxide nanoparticles for biomedical applications. Biomaterials 2005, 26:3995-4021.

19. Gossuin $Y$, Gillis $P$, Hocq A, Vuong QL, Roch A: Magnetic resonance relaxation properties of superparamagnetic particles. Wiley Interdiscip Rev Nanomed Nanobiotechnol 2009, 1:299-310.

20. Laurent S, Forge D, Port M, Roch A, Robic C, Vander Elst L, Muller RN: Magnetic iron oxide nanoparticles: synthesis, stabilization, vectorization, physicochemical characterizations, and biological applications. Chem Rev 2008, 108:2064-2110.

21. Caravan P, Farrar CT, Frullano L, Uppal R: Influence of molecular parameters and increasing magnetic field strength on relaxivity of gadolinium- and manganese-based T1 contrast agents. Contrast Media Mol Imaging 2009, 4:89-100.

22. Rozijn TH, van der Sanden BP, Heerschap A, Creyghton JH, Boveé WM: Determination of in vivo rat muscle Gd-DTPA relaxivity at $6.3 \mathrm{~T}$. Magn Reson Mater Phy 1999, 9:65-71.

23. Mazzaccara C, Labruna G, Cito G, Scarfó M, De Felice M, Pastore L, Sacchetti L: Age-related reference intervals of the main biochemical and hematological parameters in C57BL/6 J, 1299SV/EV and C3H/HeJ Mouse strains. PLOS ONE 2009, 3:e3772.

24. Mallon AM, Blake A, Hancock JM: EuroPhenome and EMPReSS: online mouse phenotyping resource. Nucleic Acids Res 2009, 36:D715-D718.

25. Telgmann L, Sperling M, Karst U: Determination of gadolinium-based MRI contrast agents in biological and environmental samples: a review. Anal Chim Acta 2013, 764:1-16.

26. Malakoutikhah M, Teixidó M, Giralt E: Shuttle-mediated drug delivery to the brain. Angew Chem Int Ed Engl 2011, 50:7998-8014.

27. Bhaskar S, Tian F, Stoeger T, Kreyling W, de la Fuente JM, Grazú V, Borm P, Estrada G, Ntziachristos V, Razansky D: Multifunctional Nanocarriers for diagnostics, drug delivery and targeted treatment across blood-brain barrier: perspectives on tracking and neuroimaging. Part Fibre Toxicol 2010, 7:3

28. Pashkunova-Martic I, Kremser C, Galanski M, Arion V, Debbage P, Jaschke W, Keppler B: Lectin-Gd-Loaded chitosan hydrogel nanoparticles: a new biospecific contrast agent for MRI. Mol Imaging Biol 2011, 13:16-24.

29. Boisselier E, Astruc D: Gold nanoparticles in nanomedicine: preparations, imaging, diagnostics, therapies and toxicity. Chem Soc Rev 2009, 38:1759-1782

30. McCarthy $R$, Weissleder R: Multifunctional magnetic nanoparticles for targeted imaging and therapy. Therapy Adv Drug Deliv 2008, 60:1241-1251. 
31. Irure Yoldi A: Paramagnetic Gd-based gold glyconanoparticles as probes for magnetic resonance imaging. (PhD Thesis). University of the Basque Country: Ciencia y Tecnologia de Polímeros; 2012.

32. de la Fuente J, Barrientos AG, Rojas TC, Rojo J, Cañada J, Fernández A, Penadés S: Gold glyconanoparticles as water-soluble polyvalent models to study carbohydrate interactions. Angew Chem Int Ed 2001, 40:2257-2261.

33. de la Fuente J, Penadés S: Glyconanoparticles: types, synthesis and applications in glycoscience, biomedicine and material science. Biochim Biophys Acta 2006, 1760:636-651.

34. Marradi M, Martín-Lomas M, Penadés S: Glyconanoparticles: polyvalent tools to study carbohydrate-based interactions. Adv Carbohydr Chem Biochem 2010, 64:211-290.

35. Rojo J, Días V, de la Fuente J, Segura I, Barrientos AG, Hans H, Riese HH, Bernad A, Penadés S: Gold Glyconanoparticles as new tools in antiadhesive therapy. ChemBioChem 2004, 5:291-297.

36. García I, Marradi M, Penadés S: Glyconanoparticles: multifunctional nanomaterials for biomedical applications. Nanomedicine UK 2010, 5:777-792.

37. Marradi MCF, García I, Penadés S: Glyconanoparticles as multifunctional and multimodal carbohydrate systems. Chem Soc Rev 2013, 42:4728-4745.

38. Barrientos AG, de la Fuente JM, Jiménez M, Solís D, Cañada FJ, Martín-Lomas M, Penadés S: Modulating glycosidase degradation and lectin recognition of gold glyconanoparticles. Carbohydr Res 2009, 344:1474-1478.

39. Hatanaka H: Transport of sugar in tumor cell membranes. Biochim Biophys Acta 1974, 355:77-104

40. Fries $P$, Runge VM, Bücker $A$, Schürholz $H$, Reith $W$, Robert $P$, Jackson $C$, Lanz T, Schneider G: Brain tumor enhancement in magnetic resonance imaging at 3 Tesla: intraindividual comparison of two high relaxivity macromolecular contrast media with a standard extracellular Gd-chelate in a rat brain tumor model. Invest Radiol 2009, 44:200-206.

41. Högemann D, Ntziachristos $V$, Josephson L, Weissleder R: High throughput magnetic resonance imaging for evaluating targeted nanoparticle probes. Bioconjugate Chem 2002, 13:116-121.

42. Swanson SD, Kukowska-Latallo JF, Patri AK, Chen C, Ge S, Cao Z, Kotlyar A East AT, Baker JR: Targeted gadolinium-loaded dendrimer nanoparticles for tumor-specific magnetic resonance contrast enhancement. Int J Nanomedicine 2008, 3:201-210.

43. Morawski AM, Winter PM, Crowder KC, Caruthers SD, Fuhrhop RW, Scott MJ, Robertson JD, Abendschein DR, Lanza GM, Wickline SA: Targeted nanoparticles for quantitative imaging of sparse molecular epitopes with MRI. Magn Reson Med 2004, 51:480-486.

44. Bridot JL, Faure AC, Laurent S, Rivière C, Billotey C, Hiba B, Janier M, Josserand V, Coll JL, Elst LV, Muller R, Roux S, Perriat P, Trillement O: Hybrid gadolinium oxide nanoparticles: multimodal contrast agents for in vivo imaging. J Am Chem Soc 2007, 129:5076-5084.

45. Flynn JR, Wang L, Gillespie DL, Stoddard GJ, Reid JK, Owens J, Ellsworth GB, Salzman KL, Kinney AY, Jensen RL: Hypoxia-regulated protein expression, patient characteristics, and preoperative imaging as predictors of survival in adults with glioblastoma multiforme. Cancer 2008, 113:1032-1042.

46. Luciani A, Olivier J-C, Clement O, Siauve N, Brillet P-Y, Bessoud B, Gazeau F, Uchegbu IF, Khan E, Frija G, Cuenod CA: Glucose-receptor MR imaging of tumors: study in mice with PEGylated paramagnetic niosomes. Radiology 2004, 231:135-142.

47. Maeda H, Wu J, Sawa T, Matsumura Y, Hori K: Tumor vascular permeability and the EPR effect in macromolecular therapeutics: a review. J Control Release 2000, 65:271-284

48. de Lussanet QG, Langereis S, Beets-Tan RG, van Genderen MH, Griffioen AW, van Engelshoven JM, Backes WH: Dynamic contrast-enhanced MR imaging kinetic parameters and molecular weight of dendritic contrast agents in tumor angiogenesis in mice. Radiology 2005, 235:65-72.

49. Balogh L, Nigavekar SS, Nair BM, Lesniak W, Zhang C, Sung LY, Kariapper MS, El-Jawahri A, Llanes M, Bolton B, Mamou F, Tan W, Hutson A, Minc L, Khan MK: Significant effect of size on the in vivo biodistribution of gold composite nanodevices in mouse tumor models. Nanomedicine 2007, 3:281-296.

50. Terentyuk GS, Maslyakova GN, Suleymanova LV, Khlebtsov BN, Kogan BY, Akchurin GG, Shantrocha AV, Maksimova IL, Khlebtsov NG, Tuchin W: Circulation and distribution of gold nanoparticles and induced alterations of tissue morphology at intravenous particle delivery. J Biophotonics 2009, 2:292-302.

51. Bomati-Miguel O, Miguel-Sancho N, Abasolo I, Candiota AP, Roca AG, Acosta M, Schwartz S Jr, Arus C, Marquina C, Martinez G, Santamaria J: Ex vivo assessment of polyol coated-iron oxide nanoparticles for MRI diagnosis applications: toxicological and MRI contrast enhancement effects. J Nanopart Res 2014, 16:2292.

52. Simoes RV, Garcia-Martin ML, Cerdan S, Arus C: Perturbation of mouse glioma MRS pattern by induced acute hyperglycemia. NMR Biomed 2008, 21:251-264.

53. Ortuño JE, Ledesma-Carbayo MJ, Simões RV, Candiota AP, Arús C, Santos A: DCE@urLAB: a dynamic contrast-enhanced MRI pharmacokinetic analysis tool for preclinical data. BMC Bioinforma 2013, 14:316.

54. Rodriguez E, Simoes RV, Roig A, Molins E, Nedelko N, Slawska-Waniewska A, Aime S, Arus C, Cabanas ME, Sanfeliu C, Cerdan S, Garcia-Martin ML: An iron-based $\mathrm{T} 1$ contrast agent made of iron-phosphate complexes: in vitro and in vivo studies. Magn Reson Mater Phy 2007, 20:27-37.

55. Robinson SP, Mclntyre DJ, Checkley D, Tessier JJ, Howe FA, Griffiths JR, Ashton SE, Ryan AJ, Blakey DC, Waterton JC: Tumour dose response to the antivascular agent ZD6126 assessed by magnetic resonance imaging. Br J Cancer 2003, 88:1592-1597.

56. Evelhoch $J$ L: Key factors in the acquisition of contrast kinetic data for oncology. J Magn Reson Imaging 1999, 10:254-259.

57. Hoffmann U, Brix G, Knopp MV, Hess T, Lorenz WJ: Pharmacokinetic mapping of the breast: a new method for dynamic MR mammography. Magn Reson Med 1995, 33:506-514.

58. Laurent $S$, Vander Elst L, Muller RN: Comparative study of the physicochemical properties of six clinical low molecular weight gadolinium contast agents. Contrast Media Mol Imaging 2006, 1:128-137.

doi:10.1186/1477-3155-12-12

Cite this article as: Candiota et al:: A new ex vivo method to evaluate the performance of candidate MRI contrast agents: a proof-of-concept study. Journal of Nanobiotechnology 2014 12:12.

\section{Submit your next manuscript to BioMed Central and take full advantage of:}

- Convenient online submission

- Thorough peer review

- No space constraints or color figure charges

- Immediate publication on acceptance

- Inclusion in PubMed, CAS, Scopus and Google Scholar

- Research which is freely available for redistribution 